\title{
Greggi e mandrie fra termalismo e profezia
}

\author{
Maddalena BASSANI \\ Dipartimento dei Beni Culturali: archeologia, storia dell'arte, \\ del cinema e della musica. Università degli Studi di Padova \\ maddalena.bassani@unipd.it
}

\section{RIASSUNTO}

A partire dal censimento dei siti termominerali di età romana attestati nella penisola italiana, l'articolo propone di interpretare alcuni esempi di ex voto animali, presenti fra i votivi di alcune stazioni curative antiche, come doni di ringraziamento per una guarigione degli animali stessi. Tale ipotesi si basa sia su dati archeologici ed epigrafici, sia su testimonianze storiche post-antiche, sia su leggende e paraetimologie, sia infine su elementi relativi alla transumanza. Si offre infine un quadro di riferimento cultuale cui inserire tali oggetti, traendo spunto dalla documentazione relativa al santuario di Apollo a Delfi.

Parole chiave: Greggi. Mandrie. Contesti termominerali. Terapia. Profezia.

\section{Flocks and herds between thermalisme and prophecy}

\begin{abstract}
Starting from the survey of thermo-mineral sites from the Roman age in the Italian peninsula, this article aims at interpreting some examples of animal ex-votos found among the offerings in some ancient healing stations, as thanksgiving for the animals' recovery. Such hypothesis is based both on archaeological and epigraphic data, and on post-ancient historical evidences, as well as on legends and pseudoetymology, and on elements pertaining to transhumance. Finally, such objects are set within a frame of reference on worship, inspired by the documentation on Apollo's shrine at Delphi.
\end{abstract}

Key words: Flocks. Herds. Thermo-mineral sites. Therapy. Prophecy.

Sumario: 1. Il quadro di riferimento. 2. La documentazione archeologica. 2.1 Veneto e Marche. 2.2 Lazio e Abruzzo. 3. La documentazione post-antica. 3.1 Testimonianze storico-letterarie. 3.2 Leggende e paraetimologie. 3.3 Terme e transumanza. 4. Due dèi pastori: Apollo e Gerione. 5. Spunti di riflessione. 
Nel settembre 2012 si è concluso un Progetto di Ricerca di Interesse Nazionale finanziato dal Ministero per l'Istruzione, l'Università e la Ricerca, che ha avuto l'obiettivo di effettuare un censimento sul territorio nazionale (con l'esclusione delle due province di Sicilia e Sardinia) di tutte le evidenze archeologiche relative allo sfruttamento delle sorgenti termominerali di età romana. ${ }^{1}$ Grazie alla realizzazione di un database, sono stati schedati circa 140 siti archeologici, sia di tipo curativo, sia di tipo cultuale; inoltre, per ognuno di essi è stata affiancata un'indagine accurata delle relative testimonianze letterarie ed epigrafiche, come pure della documentazione iconografica medievale e moderna, che poteva dare riscontro di una eventuale continuità d'uso delle stazioni curative antiche. ${ }^{2}$

Nel corso dei tre anni di lavoro, chi scrive ha seguito le problematiche relative alla documentazione cultuale di età romana, senza mancare di registrare eventuali attestazioni di epoca precedente nel caso in cui il sito abbia continuato ad essere sfruttato nei periodi di dominio romano. Fra i vari aspetti emersi, si intende qui presentare un tema poco indagato, qual è quello della presenza di votivi animali alle aquae e delle possibili implicazioni cultuali e curative che pare di intravedere dall'analisi dei manufatti.

\section{IL QUADRO DI RIFERIMENTO}

Gli studi degli ultimi decenni hanno rilevato la presenza di animali domestici fra gli ex voto di depositi votivi e di luoghi di culto concentrati per lo più nell'Italia centrale ma anche in altre province, che talora sorsero presso sorgenti d'acqua non necessariamente connotate in senso terapeutico. A partire dai contributi editi negli anni Ottanta del Novecento, infatti, si registra un'attenzione a tutti i tipi di ex voto, fra cui anche alle statuette votive di animali, ${ }^{3}$ mentre in seguito sono apparsi alcuni lavori incentrati specificamente sul rapporto fra gli animali e le pratiche religiose,${ }^{4}$ nonché sugli aspetti cerimoniali e sociali: ${ }^{5} 1$ 'attenzione degli studiosi si è concentrata sia sui resti ossei rinvenuti in contesti rituali (fosse, altari, sepolture), che rimandano evidente-

1 Il progetto "Il termalismo in età romana fra conoscenza e valorizzazione" ha coinvolto tre Università italiane (Padova: F. Ghedini, P. Zanovello, M. Annibaletto, M. Bassani; Verona: P. Basso, E. Dal Pozzolo, C. Zanetti, A. Rizzi, L. Trevisan; Genova: M. F. Petraccia, M. Tramunto), nonché il Consiglio Nazionale delle Ricerche (P. Salonia, S. Pescarin, B. Fanini, D. Ferdani), sotto il coordinamento di F. Ghedini; esso ha rappresentato un approfondimento di un altro progetto di studio relativo al sito termale antico di Montegrotto Terme (Aquae Patavinae), al quale sono stati dedicati vari articoli e tre convegni (editi in BASSANI et alii, eds., 2011, Bassani et alii, eds., 2012, e BASSANI et alii, eds., c.s.).

2 Per una prima panoramica dei dati raccolti, cf. i contributi pubblicati in BASSANI et alii, eds., c.s; per ulteriori approfondimenti cf. il volume miscellaneo ANNIBALETTO - BASSANI, eds., c.s.

3 Comella 1981, che ne dà segnalazione nella sua tavola riassuntiva; cf. inoltre BARTOLONI et alii, eds., 1989-1990; Comella - Mele, eds., 2005.

4 Cf. i vari contributi apparsi in Meniel, ed., 1989; cf. inoltre VAn ANDRINGA - LePETEZ 2003; i saggi contenuti in CAM, dir., 2007, tra cui in particolare quello di Doyen-Higuet (2007); cf. infine VolPE et alii, eds., 2010, passim.

5 Cf. Circi - Vitali, eds., 2006. 
mente a sacrifici in onore delle divinità o dei defunti, ${ }^{6}$ sia sulle statuette animali, che potevano simboleggiare, secondo la lettura più comune, un riferimento a un sacrificio o alla sostituzione del sacrificio stesso.

Tuttavia, solo occasionalmente è stata proposta una riflessione che potesse dare una spiegazione se non alternativa quanto meno ulteriore di tali oggetti: ${ }^{7}$ quella cioè che la dedica di statuette di buoi, pecore o cavalli in miniatura potesse rinviare alla richiesta di cura dell'animale, ovvero al ringraziamento per l'avvenuta guarigione della bestia.

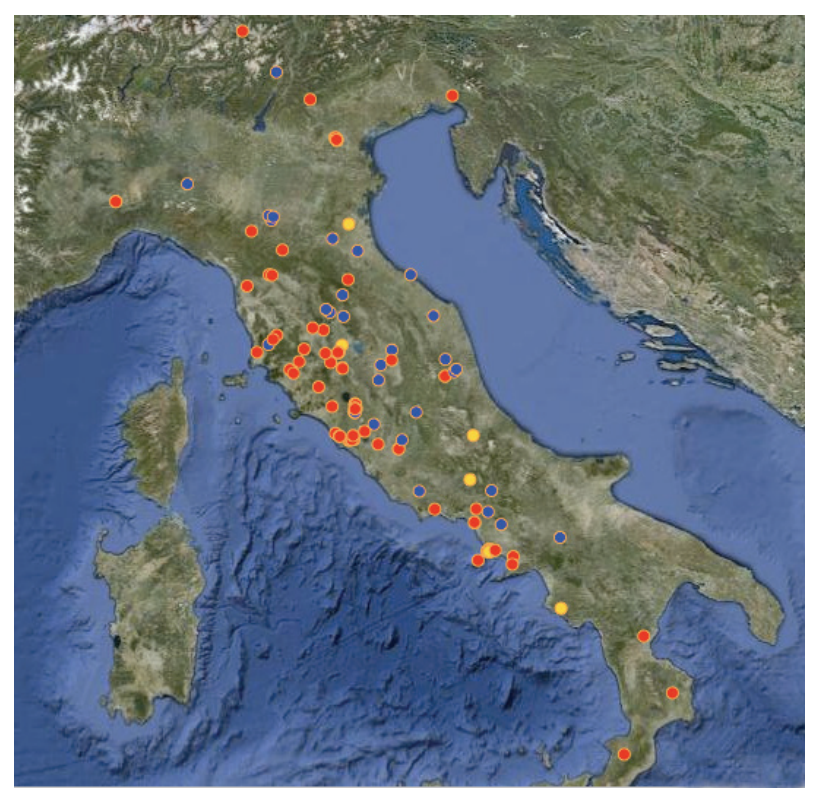

Fig. 1. Pianta distributiva delle principali stazioni termali censite (elaborazione grafica di M. Annibaletto).

Ed è proprio in tale prospettiva che possono essere analizzati alcuni dati emersi dal censimento più sopra citato, con una valenza forse ancor più incisiva: mentre in tutti i santuari, soprattutto se realizzati presso sorgenti oligominerali e dunque connotati parimenti da una sacralità dell'acqua, si poteva richiedere una generica protezione di benessere, non necessariamente una cura, alle aquae invece, e alle divinità lì presenti, si chiedeva di guarire nel caso di sterilità, di problemi gastroenterici, di patologie oculari, di ferite, ${ }^{8}$ dunque di essere risanati da un qualche male che talora poteva essere veicolato dall'animale, ${ }^{9}$ talaltra poteva colpire la bestia stessa.

6 Cf. Malnati - Gamba, eds., 2003.

7 Santillo Frizzel 2004; Santillo Frizzel 2010; Bassani 2011.

8 ZANetTi - Rizzi - Mantovanelli 2012.

9 Così Wells, a proposito dei votivi animali di Ponte di Nona (Wells 1989, 100). 
La documentazione attestata sul territorio nazionale consente di individuare quasi una trentina di contesti termominerali dislocati principalmente nel centro-Italia (Emilia Romagna, Toscana, Lazio, Campania, Abruzzo, Marche, Umbria) e nella Venetia (fig. 1), all'interno dei quali si riconoscono due tipi di indicatori: da un lato sono attestati votivi veri e propri, ${ }^{10}$ dall'altro sono presenti leggende, tradizioni ma anche toponimi che rimandano indirettamente all'utilizzo delle acque termominerali per usi veterinari. ${ }^{11}$

\section{LA DOCUMENTAZIONE ARCHEOLOGICA}

Gli oggetti dedicati nei contesti schedati, come richiesta di guarigione di animali oppure come ex voto per una salute ritrovata, corrispondono per lo più a statuette mobili, sia in bronzo che in terracotta, ma anche a monumenti con iscrizione dedicatoria oppure a oggetti più preziosi; sono databili fra l'età ellenistica e il II sec. d.C. e vengono qui considerati oltre che per regione, in senso cronologico dai più antichi ai più recenti.

\subsection{VENETO E MARCHE}

Fra gli esemplari più antichi di cui si ha riscontro materiale spiccano i votivi recuperati in due siti veneti preromani e romani, quali San Pietro Montagnon a Montegrotto Terme e Lagole di Calalzo, in provincia di Belluno: qui ancor oggi, soprattutto nel comparto euganeo, le acque termali sono sfruttate a scopi terapeutico-industriali. Proprio in quest'ultima località, in occasione di scavi effettuati fra il tardo Ottocento e il primo Novecento, è stato intercettato un laghetto di probabile età preromana, poi prosciugato in età romana, sulle sponde del quale si scoprirono migliaia di tazze, bicchieri e vasetti, oltre che un certo numero di bronzetti raffiguranti per lo più cavalli e qualche cavaliere a cavallo (fig. 2). ${ }^{12}$ Se gli studiosi hanno ricondotto le raffigurazioni dei cavalli al mito di Diomede e alla "fama" dei cavalli veneti come simboli di status,,$^{13}$ di recente si è proposto di riconoscervi, in analogia con altri simili votivi rintracciati in contesti termominerali, non tanto dei simboli di un sacrificio, quanto piuttosto animali guariti con l'immersione nelle acque termali o con l'applicazione di fanghi termali. ${ }^{14}$ Tale pratica, oltre che essere attestata in vari documenti settecenteschi locali, viene messa in atto ancor oggi comunemente, tanto da costituire un'occasione di ritrovo per appassionati di equitazione. ${ }^{15}$

Un'analoga lettura, che non ne vuole escludere altre ma che intende porsi come ulteriore ambito interpretativo, può essere proposta nel caso dei cavalli e delle raf-

\footnotetext{
10 Circa dieci contesti, senza considerare qualche altro caso incerto, recuperato nell'ultimo paragrafo.

11 Circa diciotto contesti.

12 DÄmmER, ed., 1986; i bronzetti sono datati al V-IV sec. a.C.

13 Cf. De Min 1976; DÄmmer, ed., 1986, 102.

14 BASSANi 2011.

15 Basti ricordare che il 6 luglio 2012 si è concluso ad Abano Terme, nello stesso comprensorio euganeo di Montegrotto Terme, l'equiraduno nazionale di fangoterapia per i cavalli.
} 


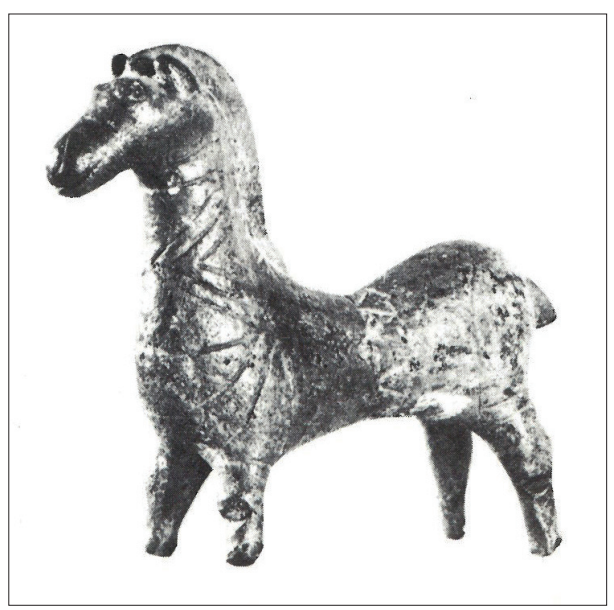

Fig. 2. Veneto, Montegrotto Terme (Padova). Cavallino in bronzo dal deposito votivo di S. Pietro Montagnon (Dämmer, ed., 1986, Taf. 13, 29g).

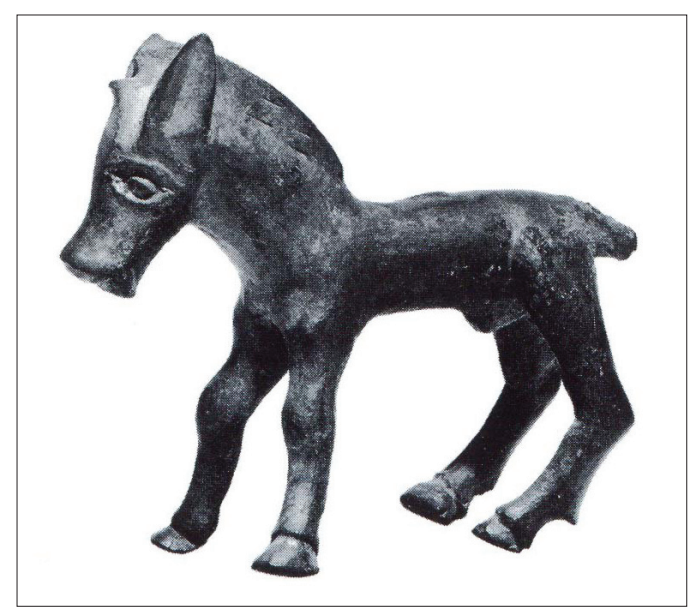

Fig. 3. Veneto, Lagole di Calalzo (Belluno). Statuetta in bronzo di cavallino votivo (Fogolari - Gambacurta, eds., 2001, 154, fig. 83).

figurazioni di cavalli nelle lamine di Lagole di Calalzo, presenti fra i votivi di un santuario dalla chiara valenza sanante (fig. 3 ), che fu frequentato dall'età preromana fino alla piena età imperiale: ${ }^{16}$ qui il rituale prevedeva anche l'impiego di simpula, per attingere e versare acqua ribollente per la presenza di gas, nei vari laghetti che dovevano caratterizzare il luogo di culto, quest'ultimo apparentemente non caratterizzato da alcuna costruzione sacra.
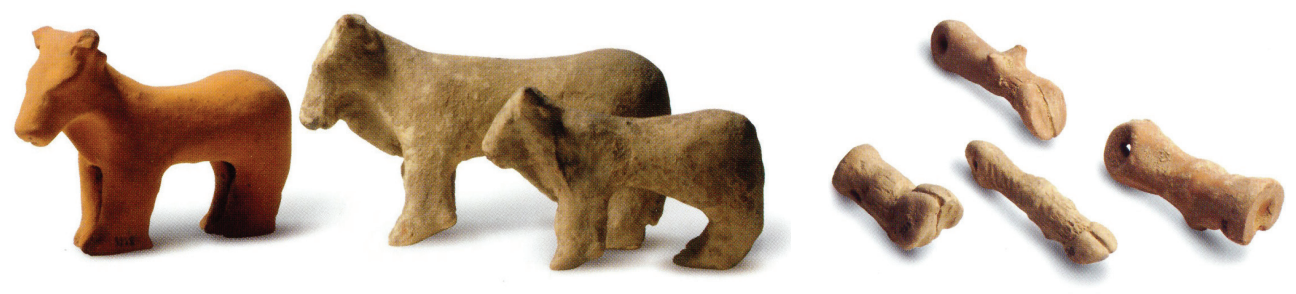

Fig. 4. Marche, Santa Veneranda (Pesaro). Statuette e zampe di animali biungulati e monoungulati dal santuario preromano-romano di Lucus Pisaurensis (Di Luca, ed., 2004, 27, fig. 32).

Anche nelle Marche si contano siti termominerali provvisti di manufatti cultuali di quadrupedi: ci si riferisce in particolare al famoso Lucus Pisaurensis, ${ }^{17}$ collocato nel

16 Fogolari - Gambacurta, eds., 2001.

17 Di LuCA, ed., 2004. 
suburbio di Pesaro, al quale pertengono quattordici cippi con dediche a varie divinità (fra cui Apollo, Feronia, Salus etc.) e soprattutto un cospicuo deposito caratterizzato da molti ex voto. Fra questi numerosi sono gli anatomici (maschere, arti, organi riproduttivi), ma vi è pure un certo numero di votivi animali (fig. 4), che riproducono sia bovini interi, sia zampe di biungulati (bovini, pecore e suini) e di monoungulati (cavalli), datati all'età tardo repubblicana. Proprio la presenza di varie zampe di bestiame da pascolo potrebbe essere un indicatore marcato della richiesta di cura per ferite o per patologie dermatologiche che potevano colpire, ieri come oggi, gli animali domestici: del resto, nelle vicinanze del Lucus sorge oggi l'impianto terapeutico di Carignano, che sfrutta sorgenti salso-bromo-iodiche con proprietà antinfiammatorie e antisettiche.

\subsection{LAZIO E ABRUZZO}

Ma la regione decisamente più ricca di manufatti "animali" è il Lazio: qui infatti si contano ben otto siti a vocazione curativa, frequentati dalla fase arcaica alla piena età imperiale, nei quali sono attestati statuette fittili o in bronzo ma anche altri oggetti che potrebbero essere interpretati in chiave "veterinaria".

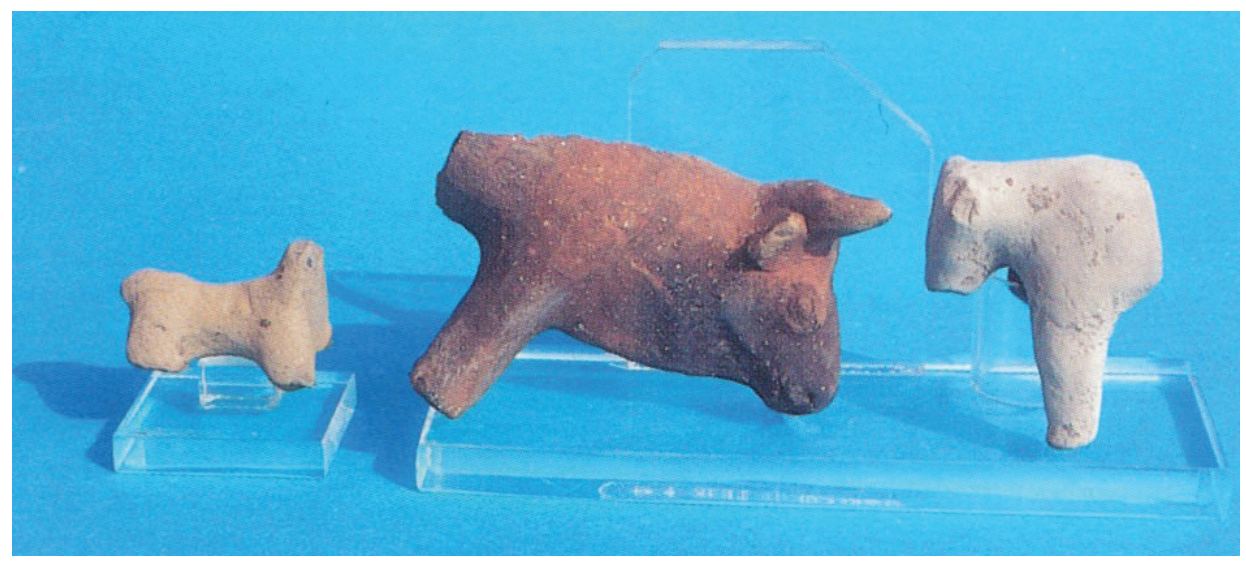

Fig. 5. Lazio, località Casale Pescarolo (Casalvieri-Frosinone). Statuette di animali da pascolo dal santuario (Bellini 2004, 104, fig. 51).

Ad esempio, esemplari fittili di buoi, tori, pecore e cavalli sono presenti in località Casale Pescarolo (in provincia di Frosinone: fig. 5) ${ }^{18}$, Tratturo Caniò (Latina) ${ }^{19} \mathrm{e}$ Ponte di Nona (nei pressi di Roma: fig. 6), ${ }^{20}$ dove esistono luoghi di culto connessi a sorgenti termominerali: in particolare, nell'ultimo sito citato si contano oltre centoquaranta esemplari animali, che vanno ad aggiungersi alle migliaia di altri anatomici

\footnotetext{
18 Bellini 2008.

19 CAssieri 2004.

20 Potter 1989.
} 


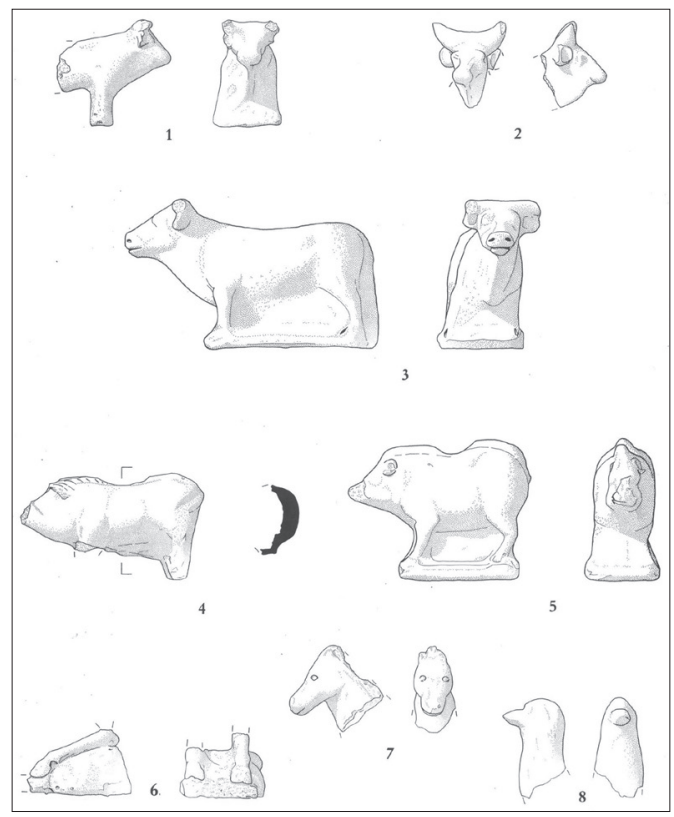

Fig. 6. Lazio, località Ponte di Nona (Roma). Disegno restitutivo di alcune statuette animali dai depositi votivi del santuario (Potter 1989, 70, fig. 68).

fittili recuperati nelle varie campagne di scavo. Si tratta di oggetti seriali, databili in un orizzonte di IV-II sec. a.C., i quali, non mostrando particolare ricercatezza stilistica, costituiscono indicatori efficaci di una committenza modesta, che traeva forse sostentamento e "ricchezza" dal possedimento di greggi e di mandrie: le tre località sono situate infatti in luoghi toccati non a caso dalla transumanza, come altri siti che si avrà modo di ricordare fra breve.

Nel Lazio, però, vi sono pure luoghi di culto connessi ad acque curative di età augustea-adrianea, dove gli oggetti sono talora più impegnativi da un punto di vista economico. In uno dei tre depositi del santuario di Gabii vi sono ancora esemplari di bovini fittili accanto a ex voto di occhi e lucerne, ${ }^{21}$ mentre alle Terme delle Zitelle, nell'area delle Aquae Sorrinenses Novenses dove le sorgenti sgorgano a 50-65 ${ }^{\circ}{ }^{22}$ è stato rintracciato un deposito apparentemente connesso non a una struttura sacra evidente, ma a piani pavimentali decorati a mosaico. Qui sono stati recuperati due frammenti in bronzo di cavallini, ${ }^{23}$ mentre un terzo esemplare, molto ossidato, è solo in parte conservato. ${ }^{24}$

21 Elvira Barba 1982, 300.

22 Chellini 2002, 121-123; Zucca 2006.

$23 \mathrm{Si}$ conservano le parti posteriori di due esemplari (uno alto $10 \mathrm{~cm}$, l'altro $0,75 \mathrm{~cm}$ ), riconducibili alla prima età imperiale.

24 L'animale è davvero piccolo: lung. $4,3 \mathrm{~cm}$. Altri oggetti da questo deposito sono alcune statuette, votivi anatomici, qualche moneta, coppette e piccoli «dadi», forse con funzione di supporto. 
Ma il caso forse più interessante ed esemplare è la nota base marmorea recuperata alle Aquae Albulae di Tivoli, ancor oggi centro curativo rinomato, sulla quale è inciso un carme in distici elegiaci che attesta la guarigione del cavallo Samis grazie a quelle acque (fig. 7). ${ }^{25} \mathrm{La}$ base reca sulla superificie superiore le impronte degli zoccoli dell'animale, che secondo uno studio recente potrebbe essere appartenuto all'imperatore Adriano ${ }^{26}$ del quale peraltro nel componimento si nominano i possedimenti della vicina Villa Adriana.

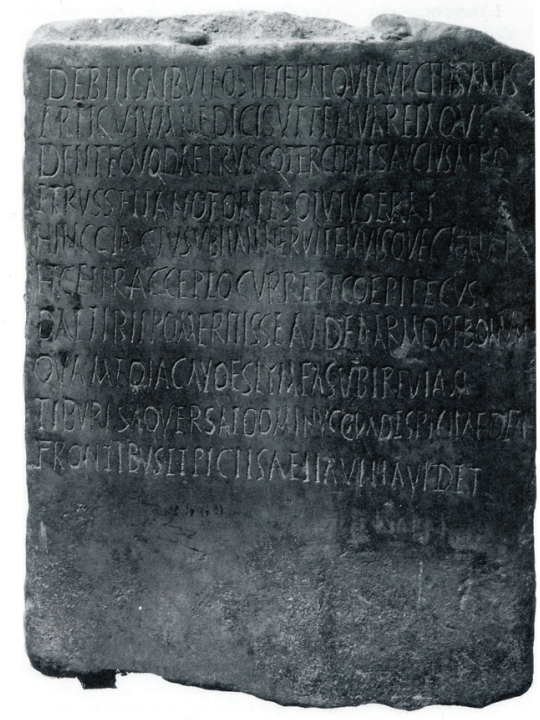

Fig. 7. Roma, Museo Nazionale Romano. La base iscritta del monumento in onore del cavallo Samis dalle Aquae Albulae (Paci 2006: 271, fig. 6).

Si tratta della più chiara attestazione di un dono fatto alle aquae per aver guarito un cavallo dalla ferita causata dalla zanna di un cinghiale, ed anzi non deve sfuggire che è Plinio a ricordarci che proprio le Aquae Albulae, classificate come sulphuratae, erano indicate specialmente per la cura delle ferite. ${ }^{27}$

Per concludere, va segnalato un ultimo ritrovamento che si configura incerto su un piano interpretativo, ma che potrebbe costituire un ulteriore elemento di valutazione: a Castel Trosino (Ascoli Piceno), dove sgorgano sorgenti sulfuree, si rinvennero nel Settecento due bacini di età romana per la raccolta dell'acqua e alcuni oggetti preziosi, tra cui "testiere" di cavalli in oro. ${ }^{28}$ Nulla ci dice che essi possano essere stati dedicati per ringraziare le aquae della guarigione di cavalli, ma possono comunque

25 Santillo Frizzel 2004.

26 GASCOU - JANON 2000, che propongono di riconoscere in Adriano anche l'autore del carme.

27 Plin Nat. Hist. 31, 6: Iuxta Romam Albulae aquae volneribus medentur, egelidae hae.

28 CONTA 1982. 
indicare una particolare riconoscenza verso quelle divinità da parte dei committenti, certamente facoltosi e legati ai propri animali in maniera particolare. ${ }^{29}$

Non va infine taciuta una leggenda antica, riferita da Rutilio Damaziano, ${ }^{30}$ che riconduceva a un animale "divino" la scoperta delle sorgenti presso le Terme Taurine (nel Lazio): un toro, che altri non era se non Giove, raspando il terreno avrebbe fatto zampillare l'acqua rivelatasi immediatemente curativa.

\section{LA DOCUMENTAZIONE POST-ANTICA}

Il censimento dei siti termominerali di età romana in Italia ha avuto anche il merito di raccogliere una cospicua mole di notizie circa il perdurare nei secoli della vocazione curativa di molte stazioni antiche. Ai fini del discorso che qui si presenta, meritano di essere ricordati tre tipi di testimonianze post-antiche: quelle che attestano una cura per gli animali attraverso bagni e fangoterapia; quelle che racchiudono nel nome un riferimento ad usi veterinari o che riportano leggende legate ad animali presso le sorgenti; e infine quelle che documentano una frequentazione dei luoghi quali punti di sosta nei percorsi della transumanza.

\subsection{TESTIMONIANZE STORICO-LETTERARIE}

All'interno del primo raggruppamento, quello appunto che raccoglie informazioni storico-letterarie su animali curati alle terme, si possono segnalare almeno tre località, ubicate lungo il versante occidentale della penisola dalla Campania all'Emilia Romagna. Proprio in questa regione, a Salvarola (Modena), sono noti alcuni impianti termali di età romana (II sec. d.C.) legati al fenomeno delle "salse", ossia a emissioni di idrocarburi liquidi e gassosi misti a fanghi, da cui si originano apparati conici (fig. 8). ${ }^{31}$ Qui per secoli i malati curarono problemi gastrenterici ed endocrinologi, come pure patologie dermatologiche attraverso le fangature ricche di zolfo, bitume e nitro; ma soprattutto ancor oggi i contadini del Modenese utilizzano questi fanghi per fare applicazioni sulle articolazioni e sui muscoli degli animali, che ne ricavano beneficio.

Nell'Ottocento, invece, a Sant'Angelo in Formis, sul Monte Tifata (Caserta), dove sorgeva un santuario preromano-romano di Diana Tifatina legato alle sorgenti termali, ${ }^{32}$ si ha testimonianza di cure veterinarie per i cavalli: lo chiariva esplicitamente G. Novi, che nella sua Memoria presentata agli Atti della Accademia Pontaniana sottolineava la necessità di prevedere, nel nuovo costituendo impianto, «una grande vasca per bagni da cavalli. Essa sarà fornita di rampe di accesso e di uscita $\mathrm{e}$

29 Un balteo in bronzo pur frammentario si conosce anche a Velia, dove però è stato trovato in giacitura secondaria, mentre ad Aosta un altro bell'esemplare è stato rinvenuto fra gli oggetti sacri di un mitreo, forse privato (FINOCCHI 2007, 26-31; BASSANI 2012a, 118, nota 22).

30 Rut. Dam. red. vv. 255 ss. Cf. Cosentino - SABbatini Tumolesi 1989, 110.

31 Guandalini 2005-2006. Si ringrazia Luciano Callegari per aver concesso la pubblicazione di una sua fotografia qui riprodotta alla fig. 8, tratta dal sito www.comune.fiorano-modenese.mo.it/turismo/salse $\% 20$ nirano/g_m/galleria_foto/galleria_foto.htm.

32 De Franciscis 1956; Quilici Gigli 2000, 46-48. 


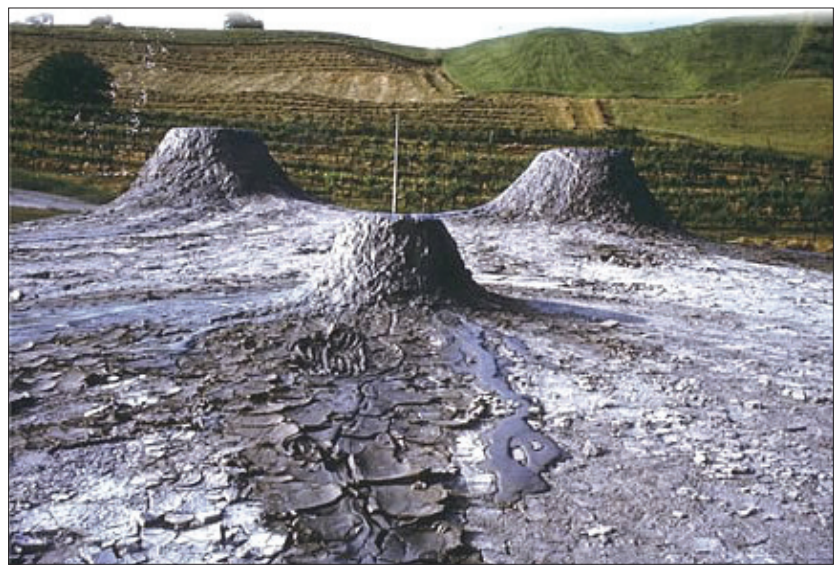

Fig. 8. Emilia Romagna, località Nirano. Veduta del fenomeno delle "salse" (foto di Luciano Callegari).

sarà coperta da tetto. Intorno intorno vi saranno delle colonnette di ferro per legarvi gli animali, durante il tempo che si bagnano». ${ }^{33}$ Più oltre, lo studioso specificava che «i cavalli, infetti da morva, farcino ${ }^{34}$ ed altre malattie contagiose, avranno una vasca speciale sulla riva sinistra del fiume ed una stalla a parte, onde non trasmettano il male ai sani od ai presi da malattie ordinarie» (fig. 9).

La testimonianza è davvero significativa, e trova riscontri in area euganea nelle note di Salvatore Mandruzzato, ${ }^{35}$ che nel tardo Settecento ricordava vasche per cavalli in varie località del comprensorio euganeo servite da percorsi minori che evidentemente non dovevano intralciare il normale transito delle persone. Ma le parole del Novi sono rilevanti anche perché confermano la pratica di isolare gli animali malati dagli altri, come già consigliava Columella nei suoi precetti sanitari. ${ }^{36}$

Altre aree per animali sono conosciute anche alle terme di Saturnia: qui le acque provenienti dalle vasche usate dai bagnanti confluivano in un bacino dove potevano immergersi le bestie, ${ }^{37}$ mentre alle Terme dell'Uncino (Torre Annunziata) si portavano i cavalli delle Scuderie del Regno delle Due Sicilie, ${ }^{38}$ perché beneficiassero della balneoterapia già nota al tempo dei romani. ${ }^{39}$

Che dunque ai bagni termali potessero andare anche gli animali domestici, è un dato che le fonti scritte di Sette e Ottocento documentano nel dettaglio. Ma doveva

\footnotetext{
33 Novi $1886,225$.

34 La morva, una malattia letale per i cavalli, si manifesta con noduli agli organi interni, alle mucose e alla pelle, mentre il farcino interessa i linfociti.

35 Riferimenti specifici in BASSANI 2011, 225-226.

36 Colum. 6, 5, 1, su cui cf. DenIs 2007, 73.

37 Chellini 2002, 127-128.

38 LANZa 1834, 100.

39 In età romana qui sorgeva una villa provvista di strutture che sfruttavano le sorgenti termali a ridosso del mare: cf. ELEFANTE 1993.
} 


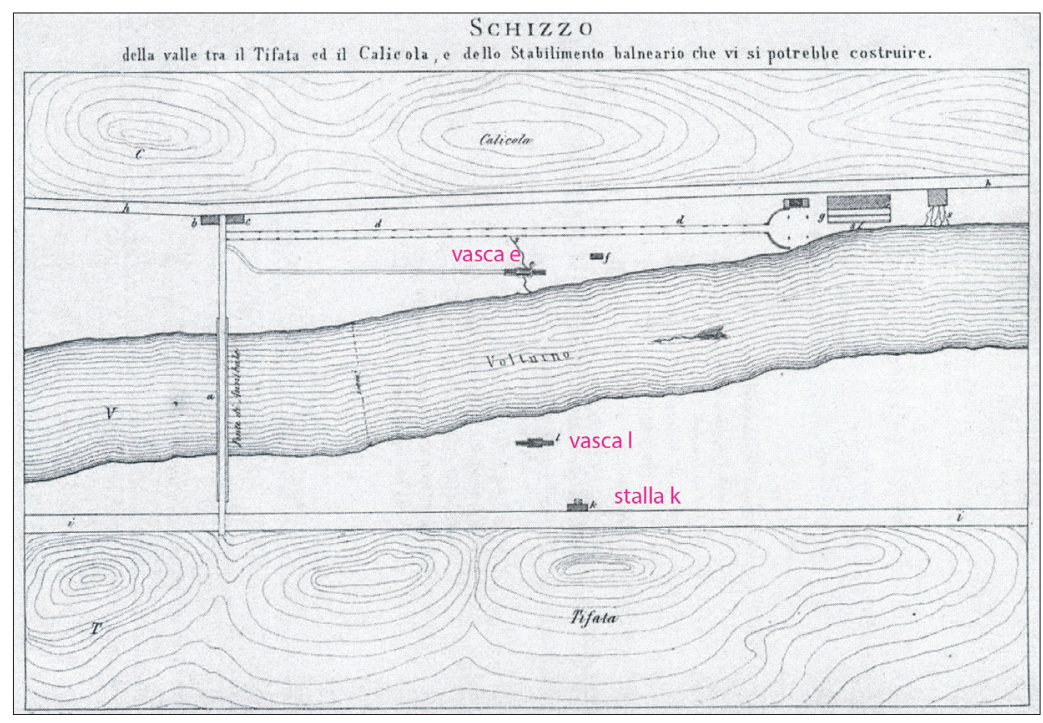

Fig. 9. Campania, Sant'Angelo in Formis, Monte Tifata (Capua). Mappa distributiva delle vasche del nuovo stabilimento termale: per i cavalli sani si doveva riservare la vasca $e$, mentre per quelli malati erano previste una vasca $l$ e una stalla $k$ al di là del fiume (Novi 1886, 257, con legenda alla pagina seguente).

essere una pratica in uso anche in precedenza: infatti nel diario di viaggio della metà del Seicento di P. Skippon a proposito dei Bagni di Abano si legge che «The water is so hot that the country people bring their hogs inter to scald off the hair. Sheep will drink of it where it is cooler, and will lick the salt». ${ }^{40}$ Dunque, se con una pratica curiosa la gente del posto conduceva alle acque caldissime le giovani pecore per lavarne il vello, le pecore stesse andavano a bere le acque fredde e leccavano i depositi di sale, elemento essenziale nell'alimentazione ovicaprina.

Ma la documentazione non si ferma qui, anzi è accresciuta da due testi cinquecenteschi che offrono ulteriori indizi al riguardo. Nel 1564 G. Falloppia descriveva una vasca per cavalli a Montegrotto Terme ${ }^{41}$ e segnalava inoltre che le sorgenti di Acqua Brandola, nel Modenese, furono scoperte accidentalmente nel 1448 in occasione di una tremenda epidemia che colpiva i buoi, mettendo a repentaglio l'economia locale. ${ }^{42}$ Si notò che i capi che andavano a bere da una precisa sorgente guarivano in breve tempo, mentre gli altri morivano: si effettuarono così analisi delle acque, che risultarono curative e vennero poi sfruttate anche per l'uomo.

Nel 1594, poi, V. Minardo ricordava che alle terme di Caldiero (Verona), ad oggi prive di indizi sicuri circa un loro sfruttamento in età romana, «Il bagno non serrato, che hora viene adoperato per li bestiami, et per quelli che pigliano il fango, è dell'i-

40 Sparti 1998, 102-103; ringrazio E. Dal Pozzolo per la segnalazione.

41 Falloppia 1564, f. $76 \mathrm{v}$.

42 Falloppia 1564, f. 38 r-v. 
stessa qualità, bontà, et virtù dell'istessa minera. La bontà di esse acque è tale, che giova quasi ad ogni creatura. La onde gli animali irrationali, come i buoi, che usano a bere di dette acque sono sempre sani, freschi, et grassi. Et essi ritrovato di molti cavalli bolsi, et inzardati con l'uso del bagno, del fango, et del bere l'acqua essersi fatti sani. Et più volte ne sono stati condotti da Mantoa, et d'altre cittadi, et risanati»». ${ }^{43}$

A quelle acque si portavano quindi buoi, cavalli e in generale «gli animali irrationali» perché ritrovassero la salute al pari degli umani; ma soprattutto dal documento si ricava che il sito era considerato un centro di cura veterinaria anche da chi abitatava a una certa distanza: alle terme ci si poteva recare seguendo anche piste e tratturi forse non sconosciuti alle vie della transumanza, come si avrà modo di proporre fra breve.

Infine, nonostante manchino elementi certi per ipotizzare trattamenti veterinari anche alle Terme del Bacucco (Viterbo), vale segnalare che qui nel Medioevo si curava con successo la scabbia, grazie alla presenza di sali di zolfo disciolti nelle sorgenti ipertermali $\left(39^{\circ}-46^{\circ}\right)$ : se in età romana era sorto un impianto ancora ben visibile da Michelangelo e da Giuliano da Sangallo, che lo ritrassero, ${ }^{44}$ si può comunque rilevare che questo tipo di malattia è la versione "umana" della "rogna", che può aggredire un intero gregge: alcuni allevatori laziali fino a pochi decenni fa erano soliti portare i loro animali perché si curassero nelle pozze termali del Viterbese (Masse di S. Sisto), situate peraltro proprio nei pressi dell'area del Bacucco.

\subsection{LEGGENDE E PARAETIMOLOGIE}

Il secondo raggruppamento che qui si propone include vari siti archeologici termali che hanno continuato ad essere frequentati nei secoli, durante i quali sono state elaborate leggende miranti ad aumentare il prestigio degli impianti: ciò avvenne attraverso mitistorie, che talora chiamarono in causa personaggi famosi dell'antichità, talaltra attestarono una funzione primaria degli animali nella scoperta delle sorgenti. Si tratta di un un aspetto curioso, al quale è dedicato uno studio interessante. ${ }^{45}$

Fra gli esempi di curiose paraetimologie, due località della Toscana e della Romagna presentano nomi "parlanti”. La stazione termale di Montecatini Terme, infatti, che conserva forse traccia di una vasca romana, ${ }^{46}$ è conosciuta oltre che come Bagno Regio, Bagno Secco, Terme Regina, anche come Bagno dei Cavalli: qui, con fangoterapia e miscelando le acque con le erbe palustri che crescevano intorno, si curavano dolori muscolari, che colpivano certamente gli uomini, ma forse anche gli animali.

Un toponimo simile si trova anche a S. Pietro in sylvis (Ravenna), la cui stazione, già frequentata in età romana, ${ }^{47}$ è nota anche come Bagnacavallo: il nome sembra derivare dalla leggenda del passaggio dell'imperatore Tiberio, il cui cavallo malato avrebbe riacquistato forza e buona salute grazie al bagno in quelle acque termali. In realtà non c'è alcuna menzione nelle fonti letterarie antiche di una sosta dell'impera-

\footnotetext{
43 Minardo 1594 , cc. 5-6.

44 Termalismo antico 1999, 49-52; Chellini 2002, 127-132.

45 Sull'argomento cf. ZANETTI c.s.

46 Chellini 2002, 182.

47 Susini 1975; Cenerini 1994.
} 
tore in questo sito, e c'è da chiedersi piuttosto se questa mitistoria non sia stata elaborata a partire da quella ben più conosciuta riferita da Svetonio: Tiberio, non ancora imperatore, si recò alle Aquae patavinae per sapere dall'oracolo di Gerione se la sua spedizione in Illiria avrebbe avuto esito positivo. ${ }^{48}$

Vi sono poi altre stazioni termali già sfruttate dai Romani che fanno risalire la loro origine a un animale domestico. Il caso più eclatante è quello dei Bagni della Porretta (Bologna), dove si conoscono tre locali forse di età romana e alcuni oggetti sporadici, tra cui la parte posteriore di un toro: ${ }^{49}$ nel XVI secolo prese piede una leggenda secondo la quale un bue si era recato a bere quelle acque per curarsi, e poiché si erano rivelate efficaci, i proprietari del luogo promossero lo sfruttamento delle sorgenti e inserirono nel loro stemma l'effigie del bue, grazie al quale era avvenuta la scoperta delle fonti curative (fig. 10).

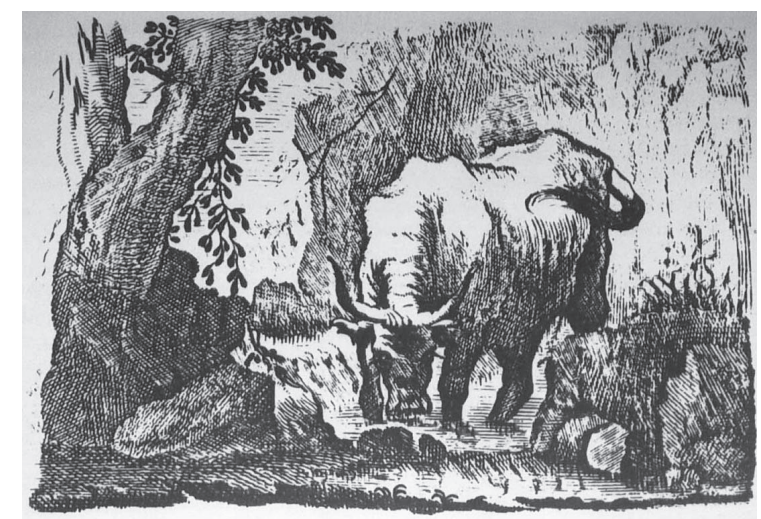

Fig. 10. Emilia Romagna, Porretta Terme (Bologna), il bue che si abbevera alle sorgenti termali (Facci, Guidanti, Zagnoni 1995, 35).

Se poi alle Terme di Comunanza, già utilizzate in età romana, una leggenda ricorda una scrofa d'oro con i suoi quattordici porcellini entro una grotta da cui sgorgava acqua terapeutica, ${ }^{50}$ al Bagno di Romagna, sede di un'importante stazione di cura e di una mansio di età tardorepubblicana-imperiale, è un cane lo scopritore delle proprietà curative di quelle acque, meglio note come Terme di S. Agnese. Si narra infatti che la giovane Agnese, ripudiata dal padre per il suo credo cristiano e gravemente malata, si fosse recata nella campagna romagnola insieme al suo cane per cercare un rifugio; fu proprio il cane ad indicare la fonte alla fanciulla, la quale, dopo aver ricevuto una pronta guarigione, iniziò il proselitismo della nuova fede. ${ }^{51}$

48 Svet. Tib. 14, 3. Ancora a questo dinasta romano è legato il nome di un altro sito termale: si tratta della Grotta del Re Tiberio, situata in provincia di Ravenna, frequentata dall'età del bronzo all'età altomedievale, dove erano presenti acque curative: cf. La grotta del Re Tiberio 1996, passim.

49 LorenZini 1974, 23; FACCI - GuIdANTI - ZAGNONI 1995.

50 Rossi Finamore - Colvetti 1976, 68.

51 Ortalli 1992, 324. 
A Comano Terme (Trentino Alto Adige), invece, la scoperta delle potenzialità curative delle acque spetta secondo una versione a un contadino, secondo un'altra ancora a un cane: nel primo caso il fattore aveva riscontrato la guarigione dalla scabbia mediante la macerazione della canapa nella sorgente (e per questo l'acqua si chiamò "acqua della rogna"); nel secondo caso era un cane malato di scabbia che andò a immergersi in quelle acque "miracolose", risultando poi perfettamente guarito e dimostrando così il valore terapeutico di quelle sorgenti. ${ }^{52}$

Gli animali, dunque, nelle tradizioni popolari erano considerati soggetti attivi e "rivelatori" del potenziale curativo di specifiche acque, indicando all'uomo i luoghi migliori in cui andare a curarsi e in cui costruire impianti terapeutici. L'animale infatti va dove l'istinto lo conduce: ma può essere anche l'uomo a portarlo in luoghi salutiferi, percorrendo sentieri che nei secoli continuano ad essere attarversati, ad esempio, dalle vie della pastorizia.

\subsection{TERME E TRANSUMANZA}

$\mathrm{Al}$ fine di inquadrare l'argomento trattato in un panorama più ampio, vale spendere qualche parola circa il rapporto che sembra profilarsi fra le stazioni di cura antiche e i percorsi delle vie della transumanza, un tema, questo, che qui si avanza in chiave problematica riservando ulteriori approfondimenti a studi futuri.

Fra le località citate, almeno tre risultano toccate da questi percorsi: Gabii, ubicata lungo i percorsi della transumanza e raffigurata nella Tabula Peutingeriana come stazione fra Roma e Preneste; Casale Pescarolo, situata lungo la direttrice interna fra Samnium e Latium, nella valle di Comino; e Tratturo Caniò, che già nel nome evoca sentieri di greggi e che era posta nella valle del Sacco presso lo snodo fra l'Appia antica e la Pedemontana. Si tratta di siti che sono considerati nella letteratura archeologica luoghi frequentanti dai pastori e forse non a caso sono tra le stazioni che conservano tracce materiali di ex voto raffiguranti pecore, buoi e cavalli.

D'altra parte, tale vocazione potrebbe aver interessato anche altre stazioni termali antiche: ad esempio, il santuario di Lagole di Calalzo, più sopra ricordato, frequentato già in età preromana da genti venete e cadorine (militari, mercanti, pastori), che percorrendo l'arteria del Piave arrivavano in direzione di Patavium con il loro bagaglio e la loro mercanzia.

Vi sono poi altri luoghi termali noti per evidenze archeologiche che risultano inseriti nelle vie percorse dalle pecore e dai buoi: tra questi si possono menzionare per primi i Bagni di Caronte, in Calabria, che sia la Tabula Peutigeriana sia alcuni testi letterari pongono in rapporto a sorgenti curative nonché ad oracoli, pur mancando evidenze strutturali certe. ${ }^{53}$ In seconda istanza vale segnalare la località di San Vittore di Cingoli, ${ }^{54}$ nelle Marche, dove sono attestati vari ambienti termali e numerosi manufatti cultuali: qui transitavano le vie armentarie, parimenti a quanto si registra nella grotta di Cesadoro (Perugia). In tale sito alcuni ritrovamenti settecenteschi han-

52 GORFER 1972.

53 De Sensi Sestito 1999.

54 SisANi 2006, 307-308. 
no conservato memoria di un nifeo-fontana di età imperiale, ma anche di un ricco deposito votivo: ${ }^{55}$ la grotta era posta lungo i percorsi delle mulattiere che transitavano fra le valli del Tevere e la zona interna del torrente Paglia, dunque anche in questo caso lungo tratturi seguiti dai pastori e dalle loro greggi. Ma vi sono ulteriori indizi di transiti di pastori umbri presso sorgenti curative, in particolare presso le terme di Santo Raggio (Assisi): qui gli animali attraversavano le colline fino ad anni recenti, toccando forse il vicino Monte Subasio, dove è noto un santuario salutifero legato ad altre sorgenti. ${ }^{56}$

Risalendo verso il nord-Italia, si segnalano poi altre due località, una in Emilia e l'altra nel Veneto. A Caverzago (Piacenza) è stato individuato un probabile santuario terapeutico in onore di Minerva, che sfruttava acque ricche di sale di Glauber (solfato di iodio discolto): si pensa che queste potessero curare problemi gastro-enterici o dermatologici, fra cui la scabbia, più volte ricordata, che si poteva trasmettere all'uomo stando in contatto con gli animali. ${ }^{57}$ A Santorso (Vicenza) le sorgenti calde sgorgano ancora in varie grotte lungo il pendio del Monte Summano, sede di un noto santuario preromano-romano connesso alla pastorizia e mèta di percorsi armetarii, tra la pianura vicentina e 1'area prealpina: ${ }^{58}$ alcune leggende elaborate fra Cinque e Seicento recano memoria di antichi riti pagani, nei quali si compivano sacrifici di montoni neri in onore del dio Summanus.

\section{DUE DÈI PASTORI: APOLLO E GERIONE}

Il panorama che sembra emergere dallo studio dei contesti termominerali antichi in Italia apre dunque a nuovi orizzonti di ricerca, che non si limitano alla mera registrazione di tipologie architettoniche balneari o alla catalogazione delle manifestazioni cultuali a queste connesse, ma offrono piuttosto vari spunti per ripensare il tema dello sfruttamento della risorsa termale sia da un punto di vista insediativo-industriale, sia da un punto di vista cultuale e religioso. ${ }^{59}$

Lasciando ad approfondimenti ulteriori l'analisi delle varie problematiche che scaturiscono dallo studio della cultualità alle terme nei suoi risvolti topografici, architettonico-tipologici nonché cronologici, può giovare in questa sede soffermarsi sul rapporto fra gli ex voto animali, presi in esame in sede iniziale, e due divinità attestate in alcuni contesti termominerali: e ciò per verificare se sia possibile inquadrare fenomenologie cultuali in apparenza seriali e simili a molte altre, in orizzonti mitologicoideali più complessi e forse più specificamente legati alle sorgenti curative e alla geotermia.

55 SENSI 2006.

56 Strazzulla 1984, 83-84. Per il momento non è chiaro a quale tipo di acque questo secondo santuario sia connesso, pertanto non è stato compreso nel censimento effettuato, ma solo segnalato nella scheda di Santo Raggio.

57 Minerva Medica 2008.

58 GamBa 2012.

59 Sono in corso di pubblicazione alcuni lavori a firma dei vari collaboratori della ricerca dedicati a questi temi nel volume AnNibaletto - Bassani, eds., c.s. (cf. supra, nota 2). 
All'interno delle realtà territoriali censite le iscrizioni e i manufatti mobili documentano, come di consueto nei santuari antichi, una pluralità di dèi che concorrevano a vari livelli alla tutela degli spazi loro pertinenti, mentre si poteva lasciare a infrastrutture il compito di marcare la separazione fra ciò che era profano e ciò che era sacro; ma non tutte le divinità erano titolari di fonti, come chiarisce Plinio il Vecchio: solo alcune potevano vantare tale specificità. ${ }^{60}$

In questa prospettiva acquistano una notevole rilevanza due soggettivi divini, Apollo e Gerione: il primo è presente nella maggior parte dei siti schedati, ${ }^{61}$ il secondo è attestato solo alle Aquae patavinae, dove peraltro svolgeva un ruolo del tutto particolare, ${ }^{62}$ anche se è nota la sua presenza pure in Sicilia, ad Agirio. ${ }^{63}$ Una considerazione di entrambi in questa sede non deve sembrare azzardata, dal momento che sia l'uno che l'altro mostrano aspetti in comune ed ambedue sembrano legati in maniera significativa alla cura e alla tutela del bestiame da pascolo.

Banalmente, li accomuna in primis una funzione benefica e salvifica: Apollo nella sua qualità di dio medico per eccellenza nonché thermios, ${ }^{64}$ Gerione nella sua "specificità" tutta patavina che lo fece divinità positiva presso le sorgenti curative. ${ }^{65}$

Entrambi sono poi, pur con le dovute differenze di "potere" e di ambiti di competenza, due dèi pastori. Se Macrobio scrive di Febo che «nelle diverse città ricorrono molti nomi che si riferiscono all'ufficio di pastore del dio [Apollo]. Perciò egli è riconosciuto come protettore e pastore per eccellenza di tutto quanto il bestiame»), ${ }^{66}$ tutta la mitologia antica fa di Gerione un pastore di mandrie, così belle e così preziose da divenire oggetto di razzia da parte di Eracle.

Ma c'è qualcosa di più che li lega in modo particolare: sia Apollo che Gerione sono titolari di oracoli legati a fenomeni "speciali" connessi al mondo sotterraneo. Il Gerione patavino era profetico grazie al manifestarsi della risalita di acque calde e ribollenti, che creavano atmosfere dense di vapori; anzi, come si è cautamente proposto di recente, ${ }^{67}$ il suo nome potrebbe evocare la sua stessa funzione oracolare. In greco, infatti, Gerioneus è un sostantivo che deriva da geryo, «faccio risonare, parlo ad alta voce, grido» e pertanto Gerione potrebbe essere, almeno in area euganea, un dio che si fa sentire dagli uomini, che parla loro "ad alta voce" forse attraverso il ribollire delle acque termali, che dà oracoli a chi glieli chiede, come al giovane Tiberio che lo interrogò prima di compiere l'impresa illirica. Ma anche Apollo è fortemente connotato come dio profetico. Nel più famoso santuario oracolare della Grecia, se-

60 Plin. ep. VIII, 8, 2-6. Per un commento a questo passo in rapporto al termalismo, cf. BASSANI 2012b.

61 Ad esempio, alle Terme di Ischia (CIL X, 6788); alle Terme di Musignano (CIL IX, 2925); alle Aquae Albulae, da cui proviene una statua del dio; alle Aquae Apollinares Veteres, dov'era un tempio apollineo; al Lucus Pisaurensis etc. La bibliografia sulla figura apollinea è sterminata: cf. quanto indicato nel Lexicon Iconographicum Mythologiae Classicae, s.v. e il recente lavoro di Ph. Monbrun relativo ai sistemi di comunicazione del dio (MonBrun 2007).

62 Per una sintesi sulla figura di Gerione in area euganea cf. i dati raccolti in BASSANI 2011, 236-238.

63 Veronese 2010.

64 Cf. CROON 1956, 205 ss.

65 Cf. Susini 1985.

66 Macr. sat. I 17, 45.

67 BASSANI 2011. 
condo Diodoro Siculo ${ }^{68}$ e Plutarco ${ }^{69}$ egli rivelava le sue profezie alla Pizia attraverso pneumata, che dal sottosuolo salivano in superficie nell'adyton del tempio e la mandavano in trance. Stando ai dati trasmessi dai due scrittori, inoltre, l'azione profetica poteva avvenire solo e soltanto se compiuti i riti di purificazione mediante le acque, che costellavano le sorgenti del santuario (fig. 11), si manifestava la volontà divina attraverso il "salto" di una capra, che veniva spruzzata con l'acqua sacra.

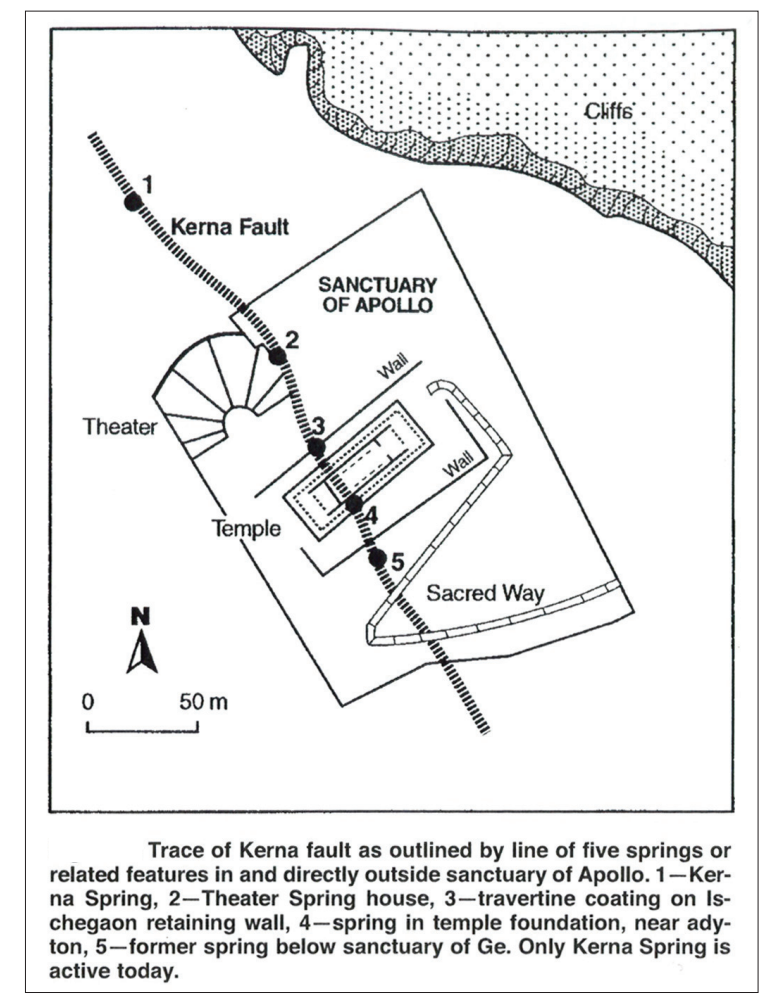

Fig. 11. Delfi, mappa con l'indicazione delle varie sorgenti nell'area del santuario di Apollo (De Boer - Hale - Chanton 2001, 708, fig. 3).

Per quanto la veridicità di tali informazioni sia stata messa in discussione all'inizio del Novecento, ${ }^{70}$ un recente articolo edito nella rivista Geology ${ }^{71}$ le ha invece recuperate e anzi avvalorate con analisi geologico-idrologiche accurate: il santuario sorse esattamente al di sopra dell'incontro di due faglie, che interagendo fra loro nei millenni hanno reso le rocce più permeabili e hanno favorito la creazione di percorsi

68 Diod. Sic. XVI, 26.

69 Plut. de defect. orac. 46-49.

70 Courby 1927, 65-66.

71 De Boer - Hale - Chanton 2001. 
per la risalita di acqua sotterranea calda e di miscugli di gas. Questi due fenomeni (fuoriuscita di acqua calda ed emissioni gassose) si manifestavano soprattutto in occasione di terremoti: ne resta traccia negli idrocarburi analizzati dagli scienziati, tra $i$ quali spicca il gas etilene dall'odore dolce, che può portare a un semplice stato di euforia se inalato in piccole quantità, ma anche a una vera e propria perdita di coscienza se non alla morte in situazioni più a rischio. Che poi con l'avvento del cristianesimo l'oracolo delfico abbia perso importanza è un dato assodato, ma come è stato suggerito da De Boer e dal suo gruppo di ricerca, è anche possibile che la concentrazione più debole dei vapori e dunque il minore potere "profetico" della Pizia, sia da imputare al venir meno di nuovi terremoti, che non produssero la consueta emissione di quello "speciale" gas narcotico.

Dunque, l'Apollo delfico è profetico grazie alla presenza di fenomeni "termali" in parte simili a quelli che caratterizzano alcuni centri termominerali peninsulari, primo fra tutti quello dell'area flegrea, sede peraltro del famoso oracolo della Sibilla Cumana, per quanto la presenza di vapori sia segnalata anche alle Terme di Agnano, a quelle di Baia, alle Stufe di Nerone, all'Ansanto e alle Grotte Parlanti, presso Monsummano. ${ }^{72}$ Ma soprattutto a Delfi le capacità oracolari di Apollo furono indiziate, secondo la leggenda, proprio dal comportamento anomalo di un branco di capre: queste, se si avvicinavano alla voragine da cui scaturivano i fumi, si mettevano a saltare in modo strano ed emettevano un verso diverso dal solito, e il loro pastore, respirando quei vapori, aveva iniziato a comportarsi in maniera inconsueta e a predire il futuro. ${ }^{73}$

Apollo, dunque, «pastore e protettore di tutto il bestiame», per dirla con Macrobio, ${ }^{74}$ aveva scelto di rivelare la sua presenza sacra e profetica attraverso un branco di capre, suggerendo agli uomini, attraverso di esse, il luogo in cui prestargli devozione e in cui poteva essere interrogato. Ma sono ancora gli animali da pascolo che nel santuario delfico sembrano svolgere un ruolo non secondario: secondo Plutarco, nel luogo sacro vi era un'area per le bestie destinate al sacrificio, che si nutrivano di erbe e germogli spuntati da quella terra alla quale, nei tempi più antichi, si era mischiato il corpo della prima Sibilla scesa dall'Elicona: «di conseguenza, i loro organi avrebbero assunto tutte le forme, i colori e le caratteristiche che producono i pronostici per il futuro» ${ }^{75}$ Questi animali, quindi, proprio perché nutritisi delle piante nate dalla terra che accolse la Sibilla, erano loro stessi portatori del messaggio divino. Non è un caso, forse, come si è ricordato più sopra, che in età storica i sacerdoti verificassero se si poteva profetizzare ricorrendo ancora a una capra: se questa si metteva a saltellare dopo che le bagnavano il pelo con acqua fredda (psychròn hydor), il dio era favorevole e la Pizia poteva rivelare le profezie; se invece non reagiva, significava che il momento non era quello giusto per conoscere la sorte.

Con tali presupposti, allora, le leggende fondative del santuario delfico mostrano in maniera evidente il ruolo centrale assunto dal bestiame sia nella fase mitica sia nella prassi rituale storica, mentre le ricerche idrogeologiche confermano l'esistenza

\footnotetext{
72 Per una panoramica su questo tipo di fenomeni geotermici cf. ANNIBALETTO c.s.

73 Diod. Sic. XVI, 26.

74 Cf. supra, nota 66.

75 Plut. de Pythiae orac. 398, 9. Sul tema cf. LinCOLN 1998, 210-211.
} 
di fenomeni geotermici particolari là dove sorse il principale santuario profetico del mondo antico.

Ovviamente con ciò non si vuole affermare che in tutti i santuari presso sorgenti termominerali in cui è attestato Apollo si debba postulare un'analoga funzione oracolare attraverso branchi di animali, piuttosto si può tentare di capire se in essi Apollo conservasse, fra le sue peculiarità, anche il suo ruolo di dio pastore di mandrie e di greggi, oltre che di dio medico per l'uomo.

D'altro canto, per il momento nulla consente di verificare un analogo utilizzo del bestiame per le azioni profetiche del Gerione patavino: troppo esigui sono per ora $\mathrm{i}$ dati a disposizione per avanzare ipotesi di qualche tipo. Tuttavia non va taciuto un confronto fra l'oracolo delle Aquae Patavinae e quello attestato dalle fonti scritte in un analogo santuario salutifero, quello di Podalirio, nell'area del Gargano: stando a Licofrone ${ }^{76}$ per ricevere responsi dal figlio di Asclepio i pellegrini si addormentavano avvolti nella pelle di pecora, secondo il rito dell'incubazione. Perché si utilizzava la pelle di pecora e non quella di un altro animale? solo perché assicurava un'adeguata protezione dal freddo a poco prezzo? oppure anche perché la pecora, come la capra, poteva fungere da tramite con il dio titolare dell'oracolo, Podalirio? Il quale, per inciso, è definito nell'Alessandra soccorritore di «uomini e greggi», dando così percezione ulteriore dello stretto legame che univa l'uomo al suo bestiame.

\section{SPUNTI DI RIFLESSIONE}

I dati proposti nelle pagine precedenti offrono, si crede, elementi utili per una riconsiderazione della tematica del termalismo antico come risorsa curativa, economica e cultuale, se pur da una prospettiva a prima vista marginale come può sembrare quella della cura degli animali. Questa è certo una problematica ancora in fieri, che potrà avvalersi di documentazioni ulteriori a conferma o a smentita delle ipotesi avanzate, anche se il testo inciso sulla base del cavallo Samis rappresenta un riscontro inequivocabile circa uno sfruttamento terapeutico-cultuale delle aquae già in età antica per usi veterinari.

Peraltro, tanto la varietà di testimonianze rinascimentali e sette-ottocentesche, quanto il perdurare di un utilizzo delle vie armentarie lungo antichi tracciati dove erano sorte stazioni curative romane, possono contribuire forse in maniera determinante all'interpretazione di oggetti apparentemente seriali e poco originali come gli ex voto animali.

Né può dirsi esaurito il problema del rapporto fra i tipi di oggetti dedicati alle aquae e i soggetti divini cui erano rivolti: la tematica è decisamente complessa e necessiterà di adeguate valutazioni che tentino di capire se sia possibile collegare i votivi alle divinità attestate nelle stazioni curative antiche. Qui sono stati scelti due dèi esemplari, pur essendoci anche altre divinità che vantano legami forti con il mondo pastorale $\mathrm{e}$ animale (fra tutti, Ercole e Artemide-Diana, presenti in alcuni siti censiti). Tuttavia,

76 Licoph. Alex. 1046-1054, ed. a cura di L. Braccesi (Roma 2004). 
essi sembrano rivelarsi particolarmente "parlanti" su un piano interpretativo, poiché racchiudono nelle loro "storie» mitologiche ed etimologiche un legame forte con l'acqua che cura e che bolle, nonché con i vapori che essa produce, acqua e vapori che scaturendo dal sottosuolo furono considerati essi stessi manifestazioni della "cosa divina" (in greco to theion è letteralmente «la divinità», ma anche «il vapore di solfo, il solfo», in latino sulphur). ${ }^{77} \mathrm{Ed}$ è tanto più interessante il legame fra queste divinità e le greggi se si considera l'ipotesi che proprio quegli animali potessero essere un tramite di Apollo e forse di Gerione per parlare agli uomini, per guarirli, per guidarli: le capre di Delfi rivelavano con i loro salti se il dio era profetico, nel Gargano le pelli di pecora servivano per sussurrare il volere di Podalirio.

E una suggestione guardare alle capre e alle pecore non solo come a "semplici" animali sacrificali ma anche come a messaggere del volere divino? Per il momento $i$ dati sono indiziari e vanno letti in chiave problematica, per quanto sarebbe sbagliato dimenticare che una tradizione descriveva la Sibilla Appenninica come una fata con gli zoccoli di capra; ${ }^{78}$ così come sarebbe sbrigativo non ricordare che ancora oggi una lana pregiata si dice "saltata" perché la pecora, immergendosi nelle pozze termali, si mette appunto a saltellare per ripulirsi dei parassiti e diventa così un capo di maggior valore. Esattamente come apparivano agli antichi i tori da sacrificare dopo che si erano bagnati nelle fonti curative del Clitumno. ${ }^{79}$

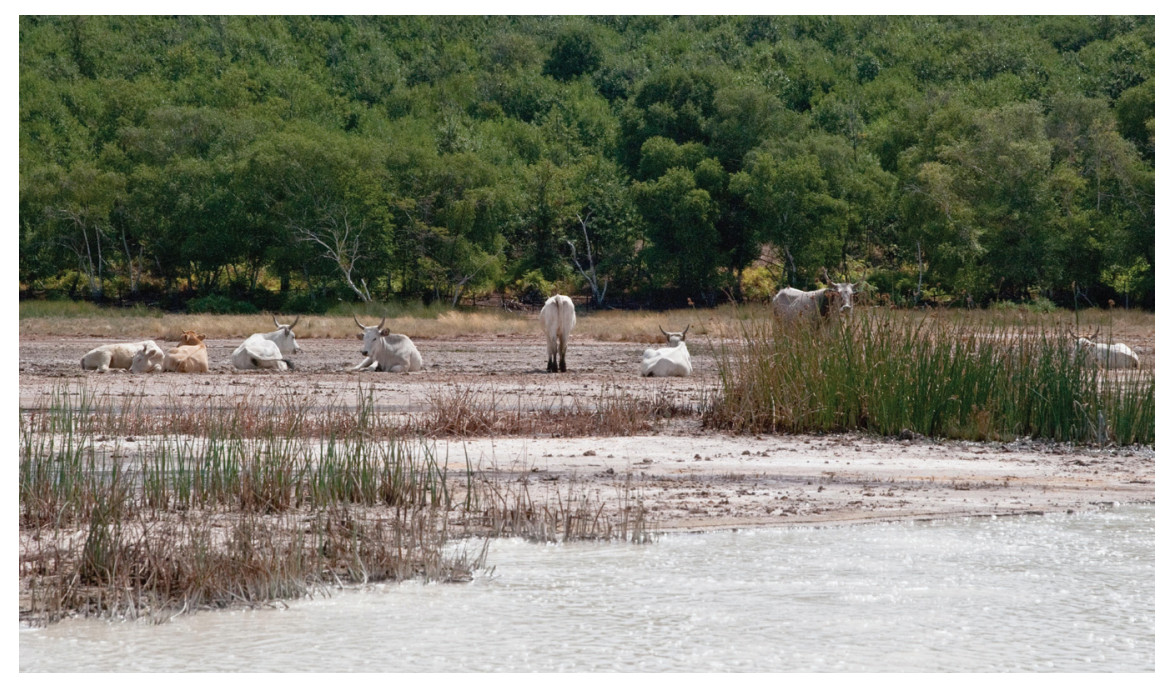

Fig. 12. Lazio, Canale Monterano, Riserva Naturale Monterano-Solfatara (Bracciano). In primo piano si nota uno dei tanti laghetti sulfurei con acque ribollenti, mentre sullo sfondo compare un branco di buoi selvatici, in riposo su uno strato di fanghi (foto dell'autrice, estate 2012).

77 Come si legge nei più comuni dizionari, ad esempio in quello di L. Rocci o di H. G. LidDELL - R. ScotT, s.v.

78 Di Modugno 1998, 809-810.

79 Verg. georg. II, 145-148; Serv. ad georg. II, 147; per un'analisi accurata cf. DuBourdieu 1997. 
In ogni caso, quella risorsa semimagica e terapeutica qual è il termalismo sembra aver curato non solo l'uomo ma anche i suoi animali: animali che gli hanno offerto nutrimento, ricchezza e forse anche un tramite con le divinità proprio attraverso quelle acque curative che per istinto vanno ancor oggi a cercare (fig. 12).

\section{BIBLIOGRAFIA}

Annibaletto, M. (c.s.): Sfruttare il termalismo. Spunti di riflessioni su tipologie architettoniche e sistemi infrastrutturali, [in] Bassani et alii, eds., c.s.

Annibaletto, M. - Bassani, M. (EDs.) (c.s.): Cura, preghiera, benessere. Le stazioni curative termali nell'Italia romana, Padova.

Bartoloni, G. - Colonna, G. - Grottarelli, G. (eds.), (1989-1990): Anathema: regime delle offerte e vita dei santuari nel Mediterraneo antico, Atti del Convegno (Roma, 15-18 giugno 1989), (=ScAnt 3-4) Roma.

BASSANI, M.

(2011): “Le terme, le mandrie e Gerione: nuove ipotesi per l'area euganea”, [in] Bassani et alii, eds., 2011, 223-243.

(2012a): “Ambienti e spazi cultuali”, [in] Annibaletto - F. Ghedini (eds.) Atria Longa Patescunt. Le forme dell'abitare nella Cisalpina romana, Saggi (=Antenor Quaderni 23.1), Roma, 111-133.

(2012b): "La schedatura dei contesti cultuali presso sorgenti termominerali. Osservazioni preliminari su aspetti strutturali e materiali”, [in] M. Bassani et alii, eds., 2012, 391-410.

BAssani, M. - Bressan, M. - Ghedini, F. (EDS.)

(2011): Aquae Patavinae. Montegrotto: le aree archeologiche e il termalismo in età antica, Atti del I Convegno Nazionale (Padova, 21-22 giugno 2010), (=Antenor Quaderni 21), Padova.

(2012): Aquae Patavinae. Montegrotto Terme e il termalismo in Italia. Aggiornamenti e nuove prospettive di valorizzazione, Atti del II Convegno Nazionale (Padova, 14-15 giugno 2011), (=Antenor Quaderni 26), Padova.

(c.s.): Aquae Salutiferae. Il termalismo antico e contemporaneo, Atti del Convegno Internazionale (Montegrotto Terme, 6-8 settembre 2012), (=Antenor Quaderni), Padova, c.s.

BeLLini, G. R.

(2004): "Il santuario di Casale Pescarolo in Valle di Comino", [in] Religio. Santuari ed ex voto nel Lazio meridionale. Atti della Giornata di studio (Terracina, 7 ottobre 2000), Terracina, 95-107.

(2008): "La piana di Alvito. Il santuario di Casale Pescarolo (Casalvieri) in Valle di Comino", [in] C. Corsi - E. Polito (eds.), Dalle sorgenti alla foce. Il bacino del Liri-Garigliano nell'antichità, culture, contatti, scambi, Atti del Convegno (Frosinone-Formia, 10-12 novembre 2005), Roma, 77-88.

Bodson, L. (1978): Hierà Zoia. Contribution à l'étude de la place de l'animal dans la religion grecque antique, Bruxelles.

CAM, M.-T. (DIR.), (2007): La médicine vétérinaire antique. Sources écrites, archéologiques, iconographiques, Actes du Colloque International (Brest, 9-11 septembre 2004), Rennes. 
CASsieri, N. (2004): "Il deposito votivo di Tratturo Caniò a Sezze", [in] Religio. Santuari ed ex voto nel Lazio meridionale, Atti della Giornata di studio (Terracina, 7 ottobre 2000), Terracina, 163-181.

Cenerini, F. (1994): "Il santuario di Bagnacavallo: documenti di religiosità sociale", [in] Storia di Bagnacavallo, a cura di Associazione «Epigraphica» (A. Calbi, G. Susini), Bologna, 97-104.

Chellini, R. (2002): Acque sorgive Salutari e Sacre in Etruria (Italiae Regio VII). Ricerche Archeologiche e di Topografia Antica (=BAR International Series 1067), Oxford.

Chirassi Colombo, I. - Seppilli, T. (EDS.), (1998): Sibille e linguaggi oracolari, Mito Storia Tradizione, Atti del Convegno Internazionale di Studi (Macerata-Norcia, 20-24 settembre 1994), Pisa-Roma.

Circi, A. - Vitali, D. (eds.), (2006): Animali tra uomini e dei. Archeozoologia del mondo preromano, Atti del convegno Internazionale (8-9 novembre 2002), (=Studi e Scavi, nuova serie 14), Bologna.

Comella, A. (1981): "Complessi votivi in Italia in epoca medio- e tardo-repubblicana", $M E$ FRA 93/2, 717-803.

Comella, A. - Mele, S. (2005), (EDS.): Depositi votivi e culti dell'Italia antica dall'età arcaica a quella tardo-repubblicana, Atti del Covegno (Perugia, 1-4 giugno 2000), Bari, 2005.

ContA, G. (1982): Il territorio di Asculum in età romana, tomo I, voll. 1-2, Pisa.

Courby, F. (1927): Topographie et architecture. La terrasse du Temple (=Fouilles de Delphes 11), Paris.

Cosentino, R. - Sabbatini Tumolesi, P. (1989): "L'edificio termale delle Aquae Caeretanae", Miscellanea ceretana 1, 95-112.

Croon, J.H. (1956): “Artemis Thermia and Apollo Thermios (with an excursus in the Oetean Heracles-cult)", Mnemosyne 9, 193-220.

Dämmer, H.-W. (ED.), (1986): San Pietro Montagnon (Montegrotto). Un santuario protostorico lacustre nel Veneto, Mainz am Rhein.

De Boer, J.Z. - Hale, J.R. - Chanton, J. (2001): "New evidence for the geological origins of the ancient Delphic oracle (Greece)", Geology 29, 707-710.

De Franciscis, A. (1956): Templum Dianae Tifatinae, Caserta.

De Min, M. (1976): “Stipe di San Pietro Montagnon”, [in] Padova preromana. Nuovo Museo Civico agli Eremitani, Catalogo della mostra (Padova, 27 giugno-15 novembre 1976), Padova, 197-218.

De Sensi Sestito, G. (1999): Tra l'Amato e il Savuto, tomo 1, Terina e il Lametino nel contesto dell'Italia antica, Soveria Mannelli.

Denis, B. (2007): “L’hygiène générale dans les écrits des agronomes latins et de Végèce”, [in] Cam, dir., 2007, 67-75.

Di LucA, M.T. (2004) (ed.): Il Lucus Pisaurensis, Pesaro.

Di Modugno, G. (1998): "Una carta delle Sibille nelle tradizioni popolari italiane”, [in] Chirassi Colombo - Seppilli, eds., 1998, 803-822.

Doyen-Higuet, A.-M. (2007): “Contribution à l'étude des textes grecs de médicine bovine”, [in] Cam, dir., 2007, 113-134.

Dubourdieu, A. (1997): "Les sources du Clitumne”, CahGlotz 8, 131-149. 
Elefante, M. (1993): "Di un opuscolo di R. Liberatore, «Delle nuove ed antiche terme di Torre Annunciata»", [in] Ercolano 1738 - 1988. 250 anni di ricerca archeologica, Atti del Convegno Internazionale (Ravello, Ercolano, Napoli, Pompei, 30 ottobre - 5 novembre 1988), Roma, 513-520.

Elvira Barba, M.A. (1982): “Terracotas votivas”, [in] M. Almagro-Gorbea (ed.), El santuario de Juno en Gabii. Excavaciones 1956-1969, Roma, 263-300.

Facci, M. - Guidanti, A. - Zagnoni, R. (1995): Le terme di Porretta nella storia e nella medicina, voll. I-II, Porretta Terme.

Falloppia, G. (1564): De medicatis aquis, Venezia.

FinOcChi, S. (2007): "Scavi e scoperte nel territorio di Aosta", [in] Da Augusta a Cesarea. Quarant'anni di ricerche, scavi e scoperte (1950-1989), Torino, 13-33.

Fogolari, G. - GambacurTa, G. (EDS.), (2001): Materiali veneti preromani e romani del santuario di Lagole di Calalzo al Museo di Pieve di Cadore, Roma.

Gamba, M. (2012): "Il Monte Summano. Un santuario sulle vie della transumanza", [in] M. S. Busana - P. Basso (eds.), A. R. Tricomi (col.), La lana nella Cisalpina romana. Economia e società, Studi in onore di S. Pesavento Mattioli, Atti del Convegno (Padova-Verona, 18-20 maggio 2011), Padova, 81-95.

GASCOU, J. - JANON, M. (2000): “Les chevaux d'Hadrien”, RAN 33, 61-68.

GASPERINI, L. (ED.), (2006): Usus veneratioque fontium. Fruizione e culto delle acque salutari nell'Italia romana, Tivoli.

Gorfer, A. (1976): Le terme di Comano nelle Giudicarie Esteriori, Calliano.

GuANDALini, F. (2006): “Approfondimenti sul fenomeno "pseudovulcanico" delle salse modenesi: estrazione del sale, uso curativo, aspetti cultuali", Ocnus 14, 275-278.

La grotta del Re Tiberio $1996=$ La grotta del Re Tiberio, [in] La collezione Scarabelli. 2. Preistoria, Imola, 1996, 419-479.

Lanza, V. (1834): "Nuove ed antiche terme di Torre Annunciata", Annali civili del Regno delle Due Sicilie IV, gennaio-aprile, 95-111.

LinCOLn, B. (1998): "La morte della Sibilla e le origini mitiche delle pratiche divinatorie”, [in] Chirassi Colombo - Seppilli, eds., 1998, 209-223.

Lorenzini, D. (1974): Guida dei Bagni della Porretta e dintorni, Bologna (ediz. anast. 1910).

Malnati, L. - GAmbA, M. (EDS.), (2003): I veneti dai bei cavalli, Treviso.

Meniel, P. (ed.), (1989): Anthropozoologica. L'animal dans les pratiques religieuses: les manifestations materielles, Actes du Colloque International (Compiegne, 11-13 novembre 1988), Compiegne.

Minardo, V. (1594): Compendio delle regole date da diversi eccellentissimi autori intorno ai Bagni di Caldiero, Verona (appresso Gerolamo Discepolo).

Minerva Medica 2008 = Minerva Medica in Valtrebbia. Scienze storiche e scienze naturali alleate per la scoperta del luogo di culto, a cura di Associazione "La Minerva" Gruppo di Ricerca Culturale - Travo, [in] Quaderni di Archeologia dell'Emilia Romagna 19.

Monbrun, Ph. (2007): Les voix d'Apollon. L'arc, la lyre et les oracles, Rennes.

Novi, G. (1886): Delle acque minerali dei monti Palombara e Tifata e dell'utilità d'una stazione balnearia presso al Ponte di Annibale, Memoria letta all'Accademia Pontaniana nella tornata del 22 giugno 1884, Napoli (rist. anast. 1979). 
Ortalli, J. (1992): “Acque e culti salutari dell’Appennino Romagnolo. Il complesso termale di Bagno di Romagna", Caesarodunum 26, 317-347.

Potter, T.W. (1989): Una stipe votiva da Ponte di Nona (=Lavori e studi di archeologia pubblicati dalla Sovrintendenza archeologica di Roma 13), Roma.

Quilici Gigli, S. (2000): "Via Dianae: appunti di topografia", [in] Campagna e paesaggio nell'Italia antica, Roma, 29-50 (ATTA 8-1999).

Rossi Finamore, E. - Colvetti, A. (1976): "La leggenda delle origini delle Terme di Bagno e gli antichi culti idrici in Romagna", StRomagn 27, 61-75.

SANTILlo Frizell, B.

(2004): "Curing the flock. The use of healing waters in Roman pastoral economy", [in] B. Santillo Frizell (cur.), Pecus. Man and animal in antiquity. Proceedings of the conference at the Swedish Institute in Rome (Rome, 9-12 September 2002), Roma, 80-93.

(2010): Lana, carne, latte. Paesaggi pastorali tra mito e realtà, Firenze.

Sensi, L. (2006): “Culto delle acque nel territorio di Tuderte”, [in] Gasperini, ed., 2006, 321-353.

SISANI, S. (2006): Umbria e Marche, Roma-Bari.

SpARTI, D.L. (1998): "Il diario di viaggio (1663-1666) di Sir Philip Skippon: Arte e Società nell'Italia del Seicento", Bollettino C.I.R.V.I. XIX, 37-38, 103-200.

Strazzulla, M.J. (1984): Assisi romana, Assisi.

Susini, G.

(1975): “Culti salutari e delle acque: materiali antichi nella Cispadana”, StRomagn 24, 321-338. (1985): "Gerione atestino", Gerión 3, 9-17.

Termalismo antico 1999 = Termalismo antico e moderno nel Lazio, Regione Lazio, Assessorato Sviluppo Economico ed Attività Produttive, Roma, 1999.

VAN ANDRINGA, W. - LePETEZ, S. (2003): "Le ossa animali nei santuari: per un'archeologia del sacrificio", [in] O. De Cazanove - J. Scheid (dirs.), Sanctuaires et sources dans l'antiquité. Le sources documentaires et leurs limites dans la description des lieux de culte, Actes de la table ronde (Napoli, 30 novembre 2001), Napoli, 85-102.

Veronese, F. (2010): “Appunti sul culto di Eracle e Gerione tra storia e archeologia”, Hesperì $26,29-46$.

Volpe, G. - Buglione, A. - De Venuto, G. (eds.), (2010): Vie degli animali, vie degli uomini. Transumanza e altri spostamenti di animali nell'Europa tardoantica e medievale, Atti del Secondo Seminario Internazionale di studi "Gli animali come cultura materiale del medioevo (Foggia, 7 ottobre 2006)”. Animals'routes, humans'routes. Transhumance and other animal movements in the medieval Europe (=Insulae Diomedeae. Collana di ricerche storiche e archeologiche 15), Bari.

Wells, C. (1989): Appendice I. Nota sugli ex-voto di Ponte di Nona, [in] Potter, 1989, 97-100.

Zanetti, C. (c.s.): "I siti termali d'Italia tra fonti letterarie e dati archeologici”, [in] Bassani et alii, eds., c.s.

Zanetti, C. - Rizzi, A. - Mantovanelli, L. (2012): “Acque e siti termali nell’Italia romana: le testimonianze degli autori antichi”, [in] Bassani et alii, eds., 2012, 365-377.

ZuccA, R. (2006): "Fruizioni idroterapiche e culti idrici nel Viterbese", [in] Gasperini, ed., 2006, 385-417. 